\title{
USO DE TRAÇADORES PARA AVALIAÇÃO DA HIDRODINÂMICA DE SISTEMAS ALAGADOS CONSTRUÍDOS OPERANDO SOB CONDIÇÕES CLIMÁTICAS TROPICAIS
}

\author{
ALISSON C. BORGES ${ }^{1}$, ANTÔNIO T. DE MATOS ${ }^{2}$, MARIA DO C. CALIJURI ${ }^{3}$, \\ GABRIEL H. H. DE OLIVEIRA ${ }^{4}$, JOÃO S. F. ROLDÃO ${ }^{5}$
}

\begin{abstract}
RESUMO: Sistemas alagados construídos (SACs) são, atualmente, importante opção para o tratamento de resíduos e controle da poluição pontual e difusa. O uso de SACs tem aumentado ano a ano, entretanto o nível de entendimento da hidrodinâmica do processo não tem crescido na mesma proporção. Traçadores fluorescentes apresentam-se como opção na determinação de curvas de distribuição de tempos de residência (DTR) e de parâmetros hidrodinâmicos, como número de dispersão e eficiência hidráulica. A pesquisa foi realizada com o objetivo de avaliar dois corantes (rodamina WT e fluoresceína sódica) na determinação das características hidrodinâmicas de SACs com escoamento subsuperficial, operando em região de clima tropical. Os tempos de residência experimentais $\left(\tau_{\mathrm{R}}\right)$ para os SACs variaram entre 4,5 e 5,0 dias, e os parâmetros de modelos teóricos foram obtidos para cada sistema, indicando dispersões muito pequenas. Os SACs, que apresentavam relação comprimento/largura (L/B) de 24/1, comportaram-se como sistemas de escoamento próximo ao pistonado. Embora a massa total adicionada não tenha sido recuperada (a adsorção é um dos mecanismos de perda), a pesquisa indicou que a rodamina WT pode ser utilizada com resultados satisfatórios na avaliação do comportamento hidrodinâmico de SACs.
\end{abstract}

PALAVRAS-CHAVE: comportamento hidrodinâmico, distribuição de tempos de residência, fluoresceína sódica, número de dispersão, rodamina WT, wetlands construídas.

\section{FLUORESCENT DYES FOR HYDRODYNAMIC EVALUATION OF CONSTRUCTED WETLANDS UNDER TROPICAL CONDITIONS}

\begin{abstract}
Nowadays, constructed wetlands (CW) systems are an important wastewater treatment option. Subsurface flow (SSF) constructed wetlands are one of the main types which are being used, and in researches, these systems dyes tracer experiments are an appropriate tool to determine residence time distribution (RDT) curves and parameters of hydrodynamic models, as a wetland dispersion numbers and hydraulic efficiency. The objectives of this paper are to evaluate and compare the performance of two fluorescent tracers (rhodamine WT and sodium fluorescein) on subsurface-flow CWs hydrodynamic characteristics determination. The cells were operated at actual hydraulic residence time $\left(\tau_{\mathrm{R}}\right)$ of a $4.5 \mathrm{~d} \sim 5.0 \mathrm{~d}$ range and the parameters of theoretical models were obtained for each system, indicating very low dispersion. The CWs with length/widths ratio equals to 24 presented as plug flow near systems. Although the recovery of total amount was small (high absorption is one of the loss mechanisms during these types of studies), rhodamine WT is a suitable tracer in mesocosms SSF constructed wetland systems of study.
\end{abstract}

KEYWORDS: hydrodynamic behavior, residence time distribution, sodium fluorescein, wetland dispersion number, rhodamine WT, constructed wetlands.

\footnotetext{
${ }^{1}$ Eng ${ }^{\circ}$ Civil, Prof. Adjunto, Departamento de Engenharia Agrícola, UFV, Viçosa - MG, Fone: (0XX31) 3899.1876, borges@ ufv.br

${ }^{2}$ Eng $^{\circ}$ Agrícola, Prof. Associado, Departamento de Engenharia Agrícola, UFV, Viçosa - MG.

${ }^{3}$ Bióloga, Profa. Titular, Departamento de Hidráulica e Saneamento, USP, São Carlos - SP.

${ }^{4}$ Eng ${ }^{0}$ Agrícola e Ambiental, Mestrando em Engenharia Agrícola, Departamento de Engenharia Agrícola, UFV, Viçosa - MG.

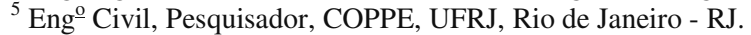

Recebido pelo Conselho Editorial em: 13-3-2008

Aprovado pelo Conselho Editorial em: 2-3-2009
} 


\section{INTRODUÇÃO}

Devido a vantagens inerentes a um processo natural, a adoção de sistemas alagados construídos (SACs) de escoamento subsuperficial, como sistemas-controle de poluição pontual e difusa, vem crescendo ano a ano. SACs (também conhecidos como wetlands construídas) têm sido usados com bons resultados no tratamento de águas residuárias, com, inclusive, a possibilidade de uso agrícola do efluente tratado (SANDRI et al., 2006). Contudo, segundo VYMAZAL (2005), o nível de entendimento dos fatores envolvidos no processo não tem aumentado na mesma proporção.

Variáveis hidráulicas têm importância fundamental no projeto e na operação satisfatória de SACs. HODGSON et al. (2004), por exemplo, lembram que todos os parâmetros usados no monitoramento da eficiência de SACs são função do tempo de residência hidráulica no sistema. Como os dados sobre o comportamento hidrodinâmico de SACs, operando em escoamento subsuperficial, ainda são escassos, as estimativas para alguns parâmetros de projeto são feitas assumindo-se o modelo idealizado de escoamento tubular pistonado.

O desvio dessa condição ideal pode ser relacionado aos processos dispersivos na unidade. A avaliação da dispersão em um SAC pode ser feita com a sua distribuição de tempos de residência (DTR). As curvas DTR podem ser obtidas a partir da técnica de estímulo e resposta com o uso de traçadores conservativos. No entanto, o ajuste dos modelos de escoamento e a obtenção dos parâmetros hidráulicos podem ser dificultados pelas condições ambientais locais e processos biológicos que ocorrem em SACs de escoamento subsuperficial. Portanto, na escolha dos traçadores a serem usados, fatores como toxicidade, reatividade, solubilidade e limite de detecção devem ser considerados (DIERBERG \& DeBUSK, 2005).

A DTR do fluido é uma função estatística que descreve a probabilidade de uma fração de partículas do fluido permanecer no volume de controle por determinado tempo, e seu uso na forma normalizada permite a comparação entre sistemas operando sob diferentes condições. A forma da obtenção da DTR, bem como os demais tópicos relativos a aspectos fundamentais no estudo da hidrodinâmica de sistemas de tratamento são apresentados nos textos de LEVENSPIEL (1974), bibliografia já consagrada sobre o tema. WERNER \& KADLEC (1996) propuseram modificações na maneira de se obter as DTRs, aplicando a teoria a situações com variação na taxa de escoamento volumétrico. A proposta mostrou-se aplicável em situações como sistemas receptores de escoamentos pluviais intensos, ocasionados em decorrência de tempestades, por exemplo.

A presente investigação foi realizada com os objetivos de avaliar o uso dos corantes fluorescentes rodamina WT e fluoresceína sódica como traçadores em SACs vegetados de escoamento subsuperficial e determinar, segundo a metodologia de WERNER \& KADLEC (1996), os parâmetros hidráulicos que definem o comportamento hidrodinâmico de SACs com diferentes inclinações, operando sob estação chuvosa, em região de clima tropical.

\section{MATERIAL E MÉTODOS}

O experimento foi realizado em SACs com escoamento subsuperficial horizontal, projetados e estudados por BRASIL (2005). As unidades foram alimentadas continuamente com água bombeada de uma das represas do ribeirão São Bartolomeu, situada na Universidade Federal de Viçosa, Minas Gerais.

Os quatro leitos constituíam-se de paredes laterais construídas em alvenaria e de uma manta de geomembrana de PVC com $0,50 \mathrm{~mm}$ de espessura, posicionada sobre o solo. Cada unidade possuía $0,35 \mathrm{~m}$ de altura, $1,0 \mathrm{~m}$ de largura e $24,0 \mathrm{~m}$ de comprimento, apresentando relação comprimento/largura (L/B) de 24/1. Como substrato, utilizou-se brita "número 0"; no entanto, as regiões de entrada de cada SAC foram preenchidas com brita "número 2" para a minimização de possíveis entupimentos. O meio-suporte preencheu as unidades até a altura de $0,30 \mathrm{~m}$, restando borda livre de 0,05 m, conforme representado no diagrama esquemático da Figura 1. 

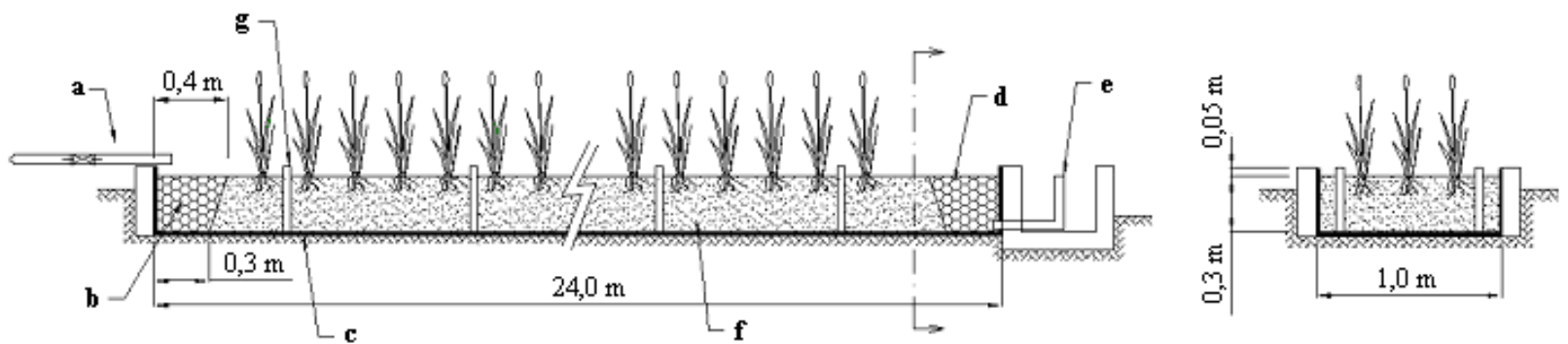

FIGURA 1. Diagrama esquemático de uma unidade do SAC: (a) dispositivo de distribuição do afluente; (b) zona de entrada; (c) geomembrana impermeável; (d) zona de saída; (e) dispositivo de extravazão para os reservatórios efluentes; (f) meio-suporte, e (g) pontos de coleta de amostras do líquido residente. Constructed wetlands cell scheme: (a) influent application; (b) inlet zone; (c) waterproofed geomembrane; (d) outlet zone; (e) outlet pipes; (f) support medium and (g) sample points .

Os leitos foram construídos com inclinações de fundo correspondentes a 0,5\% (SAC A); $1,0 \%$ (SACs B e C) e 1,5\% (SAC D). No presente estudo, foram utilizados os SACs A, C e D. À jusante dos sistemas, foram alocados reservatórios graduados (1.000 L de capacidade) para que fosse possível o cálculo diário do balanço hídrico nas unidades.

No experimento, foram utilizados corantes fluorescentes xantílicos que, devido as suas propriedades, podem ser utilizados como substâncias traçadoras: a rodamina WT e a fluoresceína sódica (também conhecida como uranina). A rodamina WT (Pylam, Inc.) foi adquirida na forma líquida concentrada (20\%), do Laboratório de Saneamento do Departamento de Hidráulica e Saneamento da EESC/USP. A fluoresceína sódica em pó foi proveniente do Laboratório de Traçadores da COPPE/UFRJ.

Os sistemas foram abastecidos com água bruta bombeada de um dos reservatórios da Universidade, e as vazões aplicadas eram equivalentes a $770 \mathrm{~L} \mathrm{~d}^{-1}$ para a unidade $\mathrm{A}, 600 \mathrm{~L} \mathrm{~d}^{-1}$ para a unidade $\mathrm{C}$ e $450 \mathrm{~L} \mathrm{~d}^{-1}$ para a unidade D. Dessa maneira, embora com volumes úteis e cargas hidráulicas distintas, todos os sistemas possuíam o mesmo tempo de residência hidráulica nominal teórico $\left(\tau_{\mathrm{N}}\right)$. Cabe ressaltar que essa previsão inicial de $\tau_{\mathrm{N}}$ foi feita sem considerar as precipitações e a perda de água por evapotranspiração nos SACs.

As soluções dos traçadores fluorescentes, com densidade igual à da fase líquida, foram injetadas na forma de pulso, na zona de entrada de cada SAC. Foram tomadas precauções para assegurar que a injeção dos corantes fosse a mais próxima possível de um sinal de entrada na forma de pulso ideal: a injeção foi feita em um curto intervalo de tempo (1 min e $30 \mathrm{~s})$ e o volume utilizado $(50 \mathrm{~L})$ foi pequeno, se comparado ao volume de líquido residente nas unidades.

Para o preparo das soluções de injeção, foram adicionados $100 \mathrm{mg}$ de cada traçador em recipientes contendo $50 \mathrm{~L}$ de água bruta. Portanto, em cada SAC, foram adicionados $100 \mathrm{mg}$ de rodamina WT e $100 \mathrm{mg}$ de fluoresceína sódica, em concentrações equivalentes a $2 \mathrm{mg} \mathrm{L}^{-1}$.

Após a adição dos corantes, foram realizadas coletas manuais de amostras simples nos pontos de saída dos SACs, para a obtenção dos valores de concentrações dos traçadores. A amostragem foi feita durante o tempo equivalente ao dobro do $\tau_{\mathrm{N}}$ previsto inicialmente. No decorrer do experimento, também foram monitoradas as variáveis $\mathrm{pH}$ e temperatura no líquido efluente, com uso de pHmetro e termômetro, respectivamente.

As amostras foram acondicionadas em frascos plásticos opacos, com capacidade para $20 \mathrm{~mL}$, protegidos da luminosidade. Em cada frasco, foi adicionada uma gota de clorofórmio, para a preservação biológica das amostras. As 73 coletas efetuadas em cada SAC foram feitas em intervalos de tempo irregulares (mínimos de 1 hora e máximos de 4 horas), considerando-se a residência teórica estimada. 
Entre duas amostragens consecutivas, mediu-se, nos reservatórios de saída, o volume de efluente gerado em cada unidade. Com esse procedimento, os valores de evapotranspiração (ET) foram estimados. Os dados obtidos por essa aproximação foram validados quando comparados aos dados climatológicos (precipitação e evaporação no tanque classe "A" e evapotranspiração de referência) da estação climatológica da Universidade Federal de Viçosa.

As concentrações efluentes dos corantes foram quantificadas por fluorescência, com auxílio de curvas de calibração, obtidas a partir de diluições das soluções adicionadas aos SACs $\left(2 \mathrm{mg} \mathrm{L}^{-1}\right)$. A fluorescência das amostras foi determinada em fluorímetro marca Turner Designs, modelo TD700 .

Conforme descrito anteriormente, para a obtenção dos parâmetros hidráulicos de interesse, foi utilizada a metodologia descrita por WERNER \& KADLEC (1996). Os parâmetros tempo de residência experimental $\left(\tau_{R}\right)$ e número de dispersão $(d)$ são decorrentes da centroide e da variância da distribuição, respectivamente. Já o parâmetro eficiência hidráulica $(\lambda)$ foi calculado a partir do tempo no qual é detectado o pico de concentração do traçador e do tempo de residência nominal (PERSSON et al., 1999).

\section{RESULTADOS E DISCUSSÃO}

A partir da medição de fluorescência nas amostras, foram obtidas as concentrações dos corantes ao longo do tempo do ensaio. Nas Figuras 2; 3 e 4, estão apresentadas as curvas de concentração versus tempo para os SACs A, C e D, respectivamente.

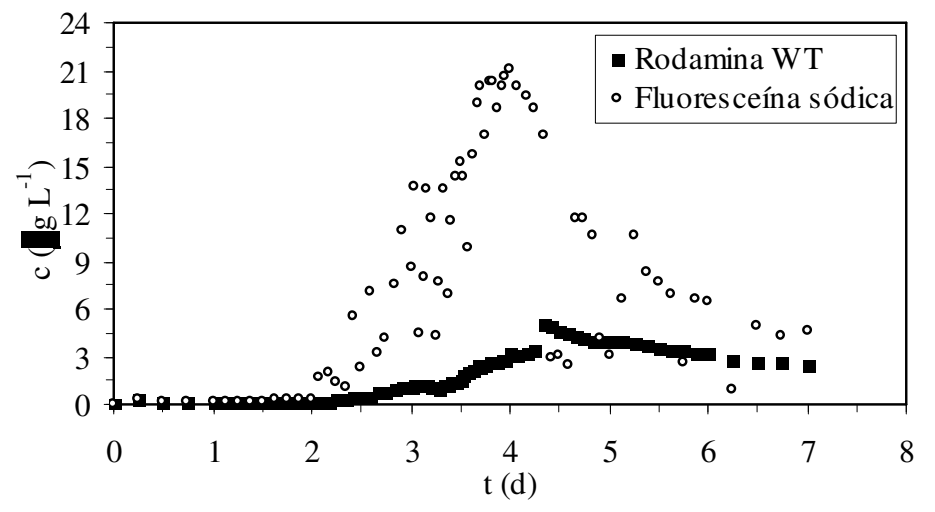

FIGURA 2. Concentrações dos traçadores fluorescentes na saída do SAC A. Fluorescent tracer concentrations in the $\mathrm{CW}$ A outlet .

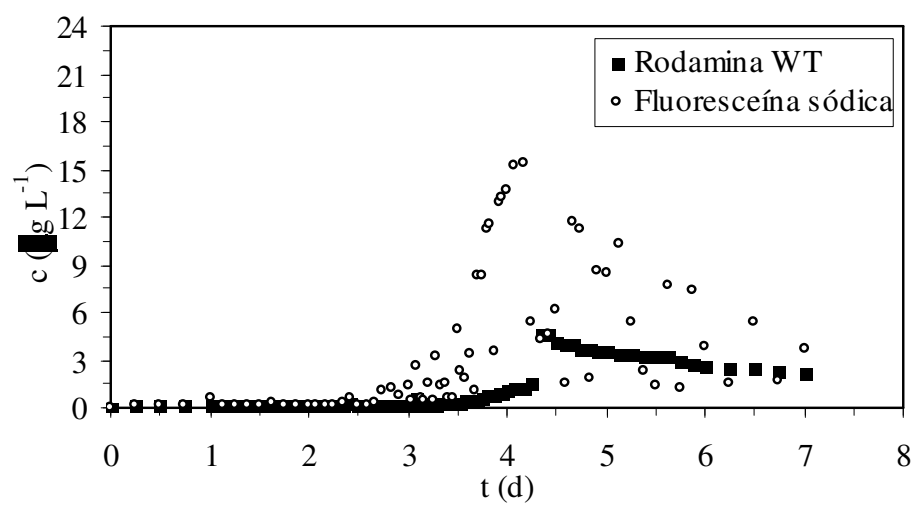

FIGURA 3. Concentrações dos traçadores fluorescentes na saída do SAC C. Fluorescent tracer concentrations in the CW C outlet . 


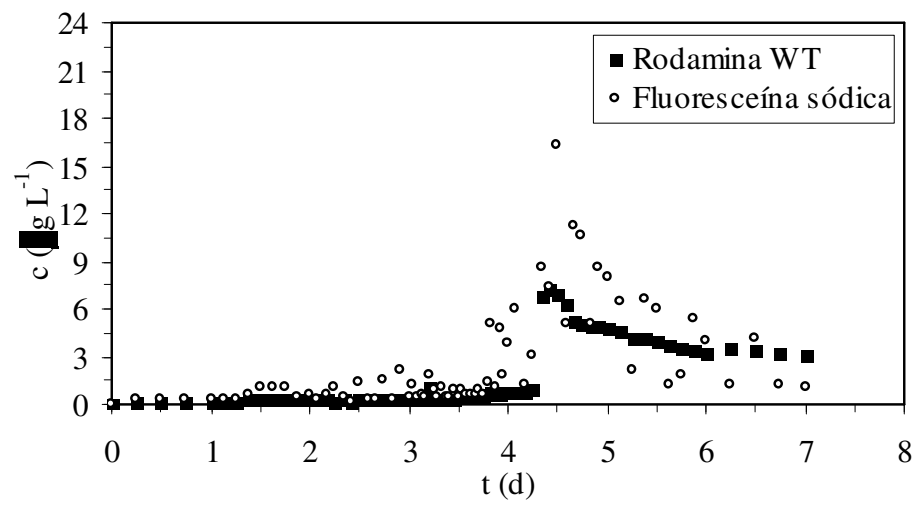

FIGURA 4. Concentrações dos traçadores fluorescentes na saída do SAC D. Fluorescent tracer concentrations in the CW D outlet .

Como pode ser observado nas Figuras 2; 3 e 4, a detecção de uranina (fluoresceína sódica) foi maior em todos os sistemas. De maneira preliminar, também podem ser citadas as longas caudas apresentadas no formato das curvas, principalmente no que se refere ao corante rodamina WT. Tal fenômeno reflete o lento sinal do traçador na saída dos sistemas.

Não se observou tempo de retardo para que valores mínimos fossem detectados na saída dos sistemas, fato que poderia ser associado às fortes chuvas que ocorreram durante o ensaio ou a condições de grande mistura. No entanto, verifica-se que os picos de passagem dos traçadores se deram após o tempo inicial estimado de 3,5 d, o que indica que, mesmo na estação chuvosa, se deve considerar a evapotranspiração da cultura. $\mathrm{O}$ rebatimento dos pontos a montante do pico, tal como feito por LIMA et al. (2004), proporcionaria melhor visualização da passagem do traçador pelo sistema, mas possivelmente subestimaria a massa total recuperada.

DIERBERG \& DeBUSK (2005) compararam o desempenho dos traçadores rodamina WT e cloreto de lítio em pequenas unidades de escoamento livre, com $1,74 \mathrm{~m}^{2}$ de área superficial. Os pesquisadores, que reportaram grandes eficiências de recuperação no ensaio principal, analisaram, paralelamente, a adsorção/dessorção e a fotólise dos compostos em sistema em batelada. A principal conclusão da pesquisa foi que reduções na recuperação dos traçadores não afetaram a precisão de obtenção dos parâmetros hidráulicos derivados da análise do método dos momentos da RTD, desde que uma resposta concentração-tempo seja discernível na curva. Tal afirmação baseia-se no fato de que as perdas (da rodamina) obedecem a reações de ordem zero e são irreversíveis.

LIN et al. (2003) reafirmam tais proposições, indicando a rodamina WT como uma substância de desempenho satisfatório no uso como traçador. Investigando um SAC de escoamento livre, em condições de campo, os pesquisadores relataram recuperação de $29 \%$ de rodamina WT e também atribuíram as perdas da substância ao mecanismo de sorção irreversível.

Embora o escoamento adotado fosse subsuperficial, há que se prever também uma possível ocorrência de fotodegradação dos corantes. SMITH \& PRETORIUS (2002) ressaltaram que a fluoresceína é degradada rapidamente, quando exposta a alta luminosidade. Outro fator importante é a perda de fluorescência devido a variações no $\mathrm{pH}$. Na faixa de valores monitorados ( $\mathrm{pH}$ equivalente a $6,3 \pm 0,2$ ), não há influência na fluorescência da rodamina WT; entretanto, na fluoresceína, pode provocar mudanças em suas propriedades espectrais (FLURY \& WAY, 2003).

O monitoramento dos volumes acumulados nos reservatórios de saída durante os intervalos das amostragens possibilitou que fossem quantificados, in loco, os valores do conjunto das componentes hidrológicas (evapotranspiração e precipitação) nos SACs. Com base no balanço hídrico de cada SAC, foi possível quantificar a vazão média e o tempo de residência teórico. Devido à diferença entre a vazão aplicada na zona de entrada e as vazões médias (obtidas somente após o 
cômputo do balanço hídrico), os tempos de residências teóricos nas unidades foram maiores que o tempo estimado inicialmente $(3,5 \mathrm{~d})$.

Com a obtenção das hidrógrafas das vazões médias durante as amostragens, foi possível a obtenção da curva de DTR, segundo proposição de WERNER \& KADLEC (1996). Na Tabela 1, são apresentados os valores dos parâmetros hidráulicos obtidos nos ensaios, cujas curvas de DTR podem ser visualizadas nas Figuras 5 e 6.

TABELA 1. Valores de tempos de residência experimentais $\left(\tau_{R}\right)$, tempos de pico $\left(t_{P}\right)$ e eficiência hidráulica $(\lambda)$ obtidos para SACs de diferentes declividades. Values of actual residence times $\left(\tau_{R}\right)$, peak tracer concentration times $\left(t_{P}\right)$ and hydraulic efficiency $(\lambda)$ obtained in CWs with different bottom slopes .

\begin{tabular}{ccccccc}
\hline \multirow{2}{*}{ SAC } & \multicolumn{2}{c}{$\tau_{\mathrm{R}}(\mathrm{d})$} & \multicolumn{2}{c}{$\mathrm{t}_{\mathrm{P}}(\mathrm{d})$} & \multicolumn{2}{c}{$\lambda$} \\
\cline { 2 - 7 } & RWT & FS & RWT & FS & RWT & FS \\
\hline A & 4,9 & 4,5 & 4,3 & 4,3 & 0,97 & 1,10 \\
C & 5,0 & 4,9 & 4,3 & 4,3 & 0,87 & 0,93 \\
D & 5,0 & 4,7 & 4,3 & 4,3 & 0,87 & 0,99 \\
\hline
\end{tabular}

RWT - rodamina WT

FS - fluoresceína sódica

Observa-se que não houve variações consideráveis em relação aos parâmetros obtidos para diferentes inclinações de fundo. Quando observados os resultados obtidos no ensaio com a rodamina WT, verifica-se que os valores de tempo de residência experimental ficaram muito próximos. A eficiência hidráulica $\lambda$ para as três unidades atingiu valores acima de 0,87 . Segundo PERSSON et al. (1999), wetlands com $\lambda$ superior a 0,75 apresentam boa condição hidráulica, e valores inferiores a 0,50 denotam $\lambda$ "pobre".

GARCÍA et al. (2004) verificaram que sistemas de escoamento subsuperficial com razão L/B maior que 4 apresentaram boas eficiências e que o parâmetro $\lambda$ apresentou correlação com a dispersão. Nos SACs com números de dispersão d inferiores a 0,08 , a $\lambda$ sempre foi maior que 0,75 .

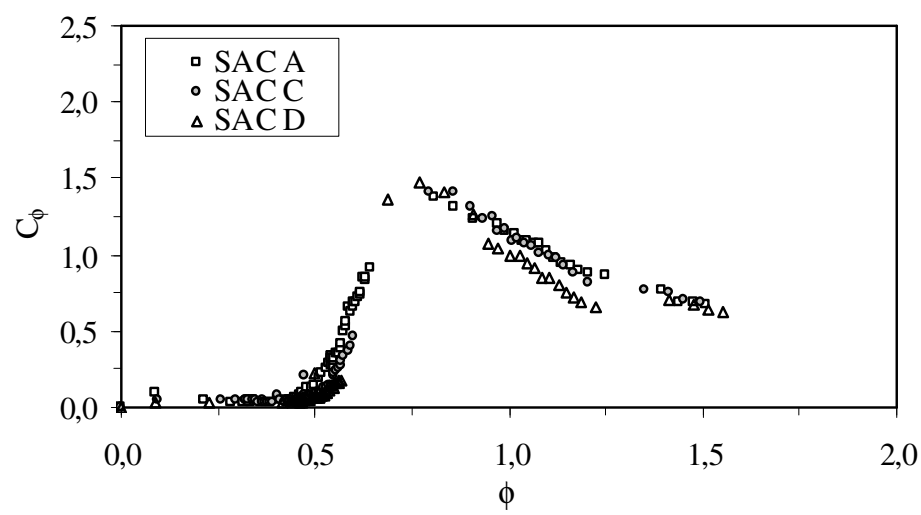

FIGURA 5. Curva adimensional de DTR referente à rodamina WT, plotada usando o tempo ponderado pelas vazões $\phi$ : comparação de SACs com diferentes inclinações de fundo e cargas hidráulicas aplicadas. Dimensionless RTDs referent to rhodamine WT, using flow weighted time $\phi$ : comparison of CWs with different bottom slopes and hydraulic load rates . 


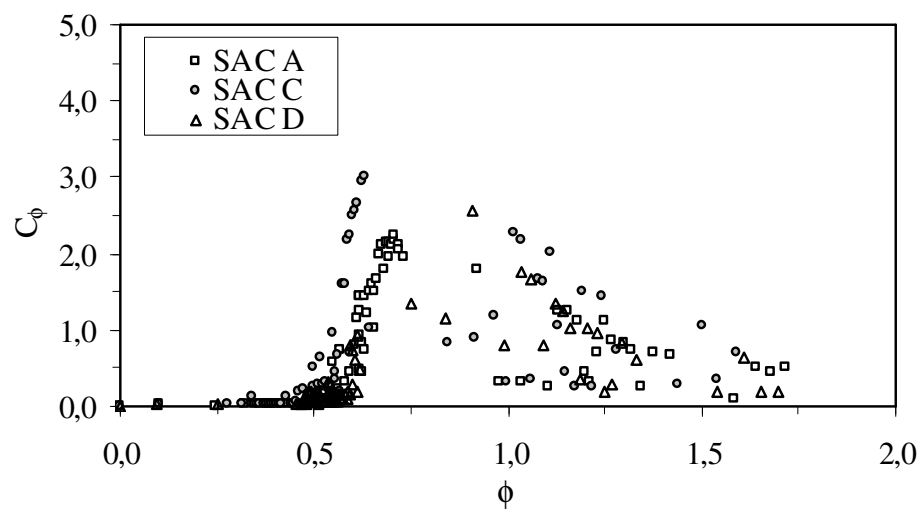

FIGURA 6. Curva adimensional de DTR referente à fluoresceína sódica, plotada usando o tempo ponderado pelas vazões $\phi$ : comparação de SACs com diferentes inclinações de fundo e cargas hidráulicas aplicadas. Dimensionless RTDs referent to sodium fluorescein, using flow weighted time $\phi$ : comparison of CWs with different bottom slopes and hydraulic load rates .

Verifica-se que o tipo de corante utilizado no ensaio influencia, sobremaneira, os resultados dos parâmetros hidráulicos. De acordo com a natureza do material traçador, resultados diferentes podem ser produzidos. Em sistemas com biofilme, fixo formado em suporte inerte como SACs de escoamento subsuperficial, a perda de massa por adsorção e difusão no meio-suporte é maior que em SACs de escoamento livre.

O tempo de surgimento dos picos de concentração foi inferior ao $\tau_{R}$ em todas as curvas; tal fenômeno indica a formação de pequenos canais preferenciais e ocorrência de regiões de estagnação. Para o corante uranina, observa-se grande variação na curva após o pico de concentração do composto. Tal fato poderia ser atribuído à recirculação do fluido no sistema, o que não corresponde ao observado para a rodamina WT, portanto supõe-se que a fluoresceína sofreu maior difusão nos poros da brita usada como substrato, já que sua massa molecular é inferior à da rodamina. Também se ressalta que a detecção desse corante em pequenas concentrações (menos que $5 \mu \mathrm{g} \mathrm{L}^{-1}$ ) é mais difícil, fato verificado quando são consideradas as curvas de calibração para detecção dos compostos.

O fenômeno de cauda observado advém, principalmente, de fenômenos de difusão e de adsorção do traçador no substrato dos sistemas, resultante da retenção superficial e contenção do produto nos poros e em várias pequenas regiões estagnadas geradas pelo suporte poroso. LIMA et al. (2004) observaram o mesmo fenômeno em sistema anaeróbio de tratamento com volume útil de aproximadamente $90 \mathrm{~L}$.

Os valores observados para os tempos de residência nominais sempre foram inferiores aos estimados utilizando-se as curvas experimentais. Embora se saiba que valores $\tau_{N} / \tau_{R}$ inferiores à unidade indiquem presença de zonas mortas no sistema, cabe ressaltar que HARDEN et al. (2003), comparando rodamina WT e fluoresceína com um traçador gasoso $\left(\mathrm{SF}_{6}\right)$ e um biológico (PRD-1), observaram que corantes fluorescentes superestimaram os tempos de residência nos sistemas de tratamento por disposição no solo (escoamento subsuperficial).

As estimativas da dispersão nos sistemas foram feitas com os números d, obtidos utilizando-se de modelos de dispersão de grande e pequena intensidade, com diferentes condições de contorno. Como pode ser observado na Tabela 2, o uso dos dois corantes resulta em estimativas ligeiramente discrepantes, contudo não houve diferença observável nos seus comportamentos, nas unidades estudadas.

As expressões analíticas disponíveis para a determinação de d diferem de acordo com as condições de contorno. O modelo de pequena intensidade não se apresenta como alternativa para a 
análise, uma vez que sua curva resultante é elaborada em condições em que $d<0,010$. Segundo KADLEC et al. (2004), os SACs horizontais geralmente apresentam-se sob condições conhecidas como recipientes fechados. Nessa situação, as características do escoamento nas seções de entrada e de saída são diferentes das que ocorrem no interior da unidade.

TABELA 2. Valores do número adimensional d, obtidos para os modelos matemáticos de dispersão de pequena e de grande intensidade. Values of dimensionless dispersion number $\mathbf{d}$, obtained by different mathematic models.

\begin{tabular}{|c|c|c|c|c|c|c|c|c|}
\hline \multirow[t]{2}{*}{ SAC } & \multicolumn{2}{|c|}{$\begin{array}{l}\text { Pequena } \\
\text { Intensidade }\end{array}$} & \multicolumn{2}{|c|}{$\begin{array}{c}\text { Grande } \\
\text { Intensidade } \\
\text { (fechado) }\end{array}$} & \multicolumn{2}{|c|}{$\begin{array}{c}\text { Grande } \\
\text { Intensidade } \\
\text { (aberto) }\end{array}$} & \multicolumn{2}{|c|}{$\begin{array}{l}\text { Grande Intensidade } \\
\text { (aberto-fechado) }\end{array}$} \\
\hline & RWT & FS & RWT & FS & RWT & FS & RWT & FS \\
\hline A & 0,039 & 0,046 & 0,041 & 0,049 & 0,034 & 0,040 & 0,037 & 0,044 \\
\hline $\mathrm{C}$ & 0,034 & 0,047 & 0,035 & 0,049 & 0,030 & 0,040 & 0,032 & 0,044 \\
\hline D & 0,043 & 0,038 & 0,045 & 0,038 & 0,038 & 0,033 & 0,041 & 0,036 \\
\hline
\end{tabular}

RWT - rodamina WT

FS -fluoresceína sódica

A despeito das diferenças observadas entre as unidades e os traçadores, verifica-se que o grau de dispersão nos SACs estudados é baixo. Os valores do número de dispersão obtidos estão inferiores à média observada na literatura. Tal fato pode ser atribuído à considerável relação L/B nas células estudadas. Na implantação dos SACs em estudo, optou-se por dividir, com muretas de concreto, uma área vegetada total de $24 \mathrm{~m}$ por $4 \mathrm{~m}$ nas quatro unidades da estação.

COTHREN et al. (2002), avaliando pequenos SACs de $4,32 \mathrm{~m}^{2}$, relataram que a dispersão observada em unidades operando em escoamento subsuperficial variou basicamente em função dos perfis de velocidades de escoamento e da razão comprimento/largura, sendo observados escoamentos com menor dispersão para SACs com razão L/B maiores que 2/1. GARCÍA et al. (2004) verificaram decréscimo nos valores de dispersão com o aumento da razão L/B. Os pesquisadores obtiveram números de dispersão na ordem de 0,050 para unidades $\left(54 \sim 56 \mathrm{~m}^{2}\right)$ com comprimento equivalente ao dobro da largura.

Os números de dispersão obtidos, na ordem de 0,040, sugerem que o movimento dos traçadores no sistema foi governado por processos advectivos e dispersivos, de maneira semelhante às observadas em outros SACs de escoamento subsuperficial, tal como observado por GARCÍA et al. (2004). Outra observação importante é que, no caso estudado, o número obtido pela equação de SPERLING (2002), que iguala d à razão $\mathrm{B} / \mathrm{L}$, é equivalente a 0,041 , o que indica haver grande correlação com os dados obtidos via perfis de DTR.

A obtenção dos parâmetros hidráulicos com confiabilidade mostra-se fator importante, aplicável no controle operacional, na verificação da adequação construtiva e na investigação de não conformidades do sistema. Ressalta-se, contudo, a necessidade da previsão de mudança no comportamento hidrodinâmico em SACs ao longo do tempo, devido a fatores como diminuição da porosidade efetiva no sistema e perdas na condutividade hidráulica nos primeiros metros das unidades.

\section{CONCLUSÕES}

Verificou-se perda dos traçadores devido, principalmente, à adsorção ao substrato e ao leito orgânico presente. Os parâmetros hidráulicos d e $\lambda$ obtidos foram maiores para fluoresceína, que se dispersou mais no meio. As curvas obtidas com o uso da rodamina WT apresentaram menor discrepância em seus valores.

As perdas de traçadores podem ser consideradas irreversíveis, e a recuperação de quantidades de traçadores inferiores às adicionadas não afetou a obtenção dos parâmetros hidráulicos. 
A proposta modificada para a obtenção de distribuição de tempos de residência para SACs, operando sob chuvas, em condições climáticas tropicais, mostrou-se satisfatória, com a obtenção das variâncias adimensionais a partir de curvas $\phi$ normalizadas em ambos os eixos.

A ocorrência de escoamentos predominantemente pistonados foi devida, basicamente, à geometria das unidades; não se observaram variações na hidrodinâmica devido à implantação de diferentes inclinações de fundo nos SACs, e, de acordo com os parâmetros obtidos, as unidades estudadas apresentaram boa eficiência hidráulica e dispersões muito pequenas $(\mathrm{d} \approx 0,040)$.

\section{REFERÊNCIAS}

BRASIL, M.S. Desempenho de sistema alagado construído para tratamento de esgoto doméstico. 2005. 160 f. Tese (Doutorado em Recursos Hídricos e Ambientais) - Universidade Federal de Viçosa, Viçosa - MG, 2005.

COTHREN, G.M.; CHEN, S.; PARDUE, J.H. Investigation of subsurface flow constructed wetlands designs. Public Works Management and Policy, Thousand Oaks, v.7, n.1, p.32-45, 2002.

DIERBERG, F.E.; DeBUSK, T. A. An evaluation of two tracers in surface-flow wetlands: rhodamine-WT and lithium. Wetlands, Lawrence, v.25, n.1, p.8-25, 2005.

FLURY, M.; WAI, N.N. Dyes as tracer for vadose zone hydrology. Reviews of Geophysics, Washington, v.41, n.1, art.1002, 2003.

GARCÍA, J.; CHIVA, J.; AGUIRRE, P.; ALVAREZ, E.; SIERRA, J.P.; MUJERIEGO, R. Hydraulic behaviour of horizontal subsurface flow constructed wetlands with different aspect ratio and granular medium size. Ecological Engineering, Amsterdam, v.23, n.3, p.177-187, 2004.

HARDEN, H.S.; CHANTON, J.P.; ROSE, J.B.; JOHN, D.E.; HOOKS, M.E. Comparison of sulfur hexafluoride, fluorescein and rhodamine dyes and the bacteriophage PRD-1 in tracing subsurface flow. Journal of Hydrology, Amsterdam, v.277, n.1-2, p.100-115, 2003.

HODGSON, C.J.; PERKINS, J.; LABADZ, J.C. The use of microbial tracers to monitor seasonal variations in effluent retention in a constructed wetland. Water Research, Oxford, v.38, n.18, p.3.833-3.844, 2004.

KADLEC, R.H.; WALLACE, S.; VYMAZAL, J. Treatment wetlands. $2^{\text {nd }}$ ed. Boca Raton: CRCLewis, 2004. 800 p.

LEVENSPIEL, O. Engenharia das reações químicas: cálculo de reatores. São Paulo: Edgard Blucher, 1974. $211 \mathrm{p}$.

LIMA, C.A.A.L.; SILVEIRA, A.; ZAIAT, M. Considerações sobre ensaios hidrodinâmicos em reator anaeróbio horizontal de leito fixo (RAHLF) tratando esgoto sanitário. In: SIMPÓSIO BRASILEIRO DE ENGENHARIA AMBIENTAL, 3., 2004, Brasília. Anais... Brasília: UCB, 2004. 1 CD-ROM.

LIN, A.Y.C.; DEBROUX, J.F.; CUNNINGHAM, J.A.; REINHARD, M. Comparison of rhodamine WT and bromide in the determination of hydraulic characteristics of constructed wetland. Ecological Engineering, Amsterdam, v.20, n.1, p.75-88, 2003.

PERSSON, J.; SOMES, N.L.G.; WONG, T.H.F. Hydraulics efficiency of constructed wetlands and ponds. Water Science and Technology, Londres, v.40, n.3, p.291-300, 1999.

SANDRI, D.; MATSURA, E.E.; TESTEZLAF, R. Teor de nutrientes na alface irrigada com água residuária aplicada por sistemas de irrigação. Engenharia Agrícola, Jaboticabal, v.26, n.1, p.45-57, 2006.

SMITH, S.A.; PRETORIUS, W.A. The conservative behavior of fluorescein. Water SA, Pretoria, v.28, n.4, p.203-206, 2002. 
SPERLING, M. Von. Princípios de tratamento biológico de águas residuárias: lagoas de estabilização. 2.ed. Belo Horizonte: DESA/UFMG, 2002. v.3, 196 p.

VYMAZAL, J. Constructed wetlands for wastewater treatment. Ecological Engineering, Amsterdam, v.25, n.5, p.475-477, 2005.

WERNER, T.M.; KADLEC, R.H. Application of residence time distributions to stormwater treatment systems. Ecological Engineering, Amsterdam, v.7, n.3, p.213-234, 1996. 